\title{
Phase Transition Modeling for Cavitating Flows via a Hybrid Explicit Residual Distribution Scheme
}

\author{
Paola Bacigaluppi, R'emi Abgrall \\ Institut f ur Mathematik, Universit"at Z"urich, Winterthurerstrasse 190, CH-8057 Z"urich, Switzerland \\ Paola.bacigaluppi@math.uzh.ch; Remi.abgrall@math.uzh.ch
}

\section{Extended Abstract}

The aim of this work is the development of a fully explicit high-order scheme in the framework of compressible multiphase flows with heat and mass transfer. The study considers the five equation model given in [1], which is obtained from the formal limit [2] of the Baer and Nunziato model [3] when the relaxation parameters tend simultaneously to infinity. This model consists in one mass conservation law for each phase, one conservation law for the momentum and one for the energy for the phase-mixture coupled to a transport equation for the volume fraction of one phase1. This last equation is in a nonconservative form, leading to several questions on how to define and approximate its solutions. As suggested in [4,5], the model is split into a hyperbolic and a phase transition contribution. The adopted scheme for the hyperbolic terms, consists in a predictor-corrector scheme. Once the hyperbolic part has been computed, a thermo-chemical relaxation allows to compute the heat and mass transfer terms as in [5]. This terms represent the phase transition contribution of the model.

For the hyperbolic part of the problem, following [6], the study considers one prediction and one correction step, adopting for the space discretization a residual based distribution scheme. This formulation allows seeing the component of the discretization as an error between two different approximations. Here, the residual is computed considering a Lax Friedrich's scheme [6]. In order to construct a stable and non-oscillating high-order accurate approximation across discontinuities, the residual, maintaining the consistency requirement, is distributed to each node belonging to a cell via a limiter. The last is designed similarly to a positive stream-wise invariant with a filtering term, using its eigenforms instead of the residual. The heat and mass transfer terms are coupled to the hyperbolic solution as suggested in [4] and the admissibility procedure, as in [8], is added to ensure the positivity of the solution.

With respect to other contributions in the area of phase transition models for cavitating flows, we investigate a method that provides mesh convergence to the exact solutions, where the studied non-conservative system is associated to consistent jump relations and follows the idea of [7]. This numerical methodology can be easily extended to unstructured meshes and to the six and seven equation models. Finally, the dodecane liquid-vapour shock tube benchmark $[4,5]$ has shown very good overlaps with the expected solutions and has assessed the robustness and accuracy of the presented method.

\section{References}

[1] A. K. Kapila, R. Menikoff, J. B. Bdzil, S. F. Son, D.S. Stewart, "Two-phase modeling of deflagration-to-detonation transition in granular materials: Reduced equations," Phys. Fluids, vol. 13, pp. 30023024, 2001.

[2] A. Murrone and H. Guillard, "A five equation reduced model for compressible two phase flow problems," J. Comput. Phys., vol. 202, pp. 664-698, 2005.

[3] M. R. Baer and J. W. Nunziato, "A two-phase mixture theory for the deflagration-to-detonation transition (DDT) in reactive granular materials," Int. J. Multiph. Flows, vol. 12, pp. 861-889, 1986.

[4] R. Saurel, F. Petitpas and R. Abgrall, "Modelling phase transition in metastable liquids. application to cavitating and flashing flows," J. Fluids Mech., vol. 607, pp. 313350, 2008.

[5] M. G. Rodio and R. Abgrall, "An innovative phase transition modeling for reproducing cavitation through a fiveequation model and theoretical generalization to six and seven-equation models," Int. J. Heat and Mass Trans., vol. 89, pp. 1386-13140, 2015.

[6] M. Ricchiuto and R. Abgrall, "Explicit Runge-Kutta residual distribution schemes for time dependent problems: second order case," J. Comput. Phys., vol. 229, pp. 5653-5691, 2010. 
[7] R. Abgrall and H. Kumar, "Numerical approximation of a compressible multiphase system," Comm. Comput. Phys., vol. 15, pp. 1237-1265, 2014.

[8] M. Pelanti and K. M. Shyue, "A mixture-energy-consistent six-equation two-phase numerical model for fluids with interfaces, cavitation and evaporation waves,” J. Comput. Phys., vol. 259, pp. 331357, 2014. 